\title{
əIn Situ Underwater Average Flow Velocity Estimation Using a Low-Cost Video Velocimeter $\mathscr{C}$
}

\author{
Pichaya Lertvilai, ${ }^{\mathrm{a}}$ PAUl L.D. Roberts, ${ }^{\mathrm{b}}$ AND Jules S. JAFFe ${ }^{\mathrm{a}}$ \\ ${ }^{a}$ Scripps Institution of Oceanography, University of California, San Diego, La Jolla, California \\ ${ }^{\mathrm{b}}$ Monterey Bay Aquarium Research Institute, Moss Landing, California
}

(Manuscript received 23 July 2020, in final form 25 March 2021)

\begin{abstract}
The development of a low-cost Video Velocimeter (VIV) to estimate underwater bulk flow velocity is described. The instrument utilizes a simplified particle image correlation technique to reconstruct an average flow velocity vector from video recordings of ambient particles. The VIV uses a single camera with a set of mirrors that splits the view into two stereoscopic views, allowing estimation of the flow velocity vector. The VIV was validated in a controlled flume using ambient seawater, and subsequently field tested together with an acoustic Doppler velocimeter with both mounted close to the coastal seafloor. When used in nonturbulent flow, the instrument can estimate mean flow velocity parallel to the front face of the instrument with root-mean-squared errors of the main flow within $10 \%$ of the $\pm 20 \mathrm{~cm} \mathrm{~s}^{-1}$ measurement range when compared to an acoustic Doppler velocimeter (ADV). The predominant feature of the VIV is that it is a cost-effective method to estimate flow velocity in complex benthic habitats where velocity parallel to the sea floor is of interest.
\end{abstract}

KEYWORDS: In situ oceanic observations; Instrumentation/sensors; Measurements

\section{Introduction}

Since the beginnings of oceanography, the utility of instruments to measure ocean currents has been an important aspect of studying the ocean. The ocean currents determine the fate of both inanimate particles and many living organisms in marine environments. Nutrients from rivers, groundwater and atmosphere are distributed and transported offshore by coastal currents (Jickells 1998) and can eventually be carried back to the coasts by upwelling currents (Martin and Richards 2001). The survival of many organisms, especially suspension feeders, depend on ocean currents to provide nutrients (Hill et al. 1992) and to support larval transport to suitable settlement areas (Pineda et al. 2011). Even small-scale turbulence can have profound effects on marine organisms by determining predatorprey contact rates (Rothschild and Osborn 1988) and by forming nutrient patches for foraging (Davis et al. 1991). As a result, the advancement of instruments to measure ocean currents has been an active development area throughout the history of oceanography.

The earliest marine current velocity sensors were based on the rotation of a propeller-like device around a shaft, such as the Ekman current meter (Ekman 1932), the Roberts current meter (Knauss 1959), and the Savonius current meter (Weller and Davis 1980). Electromagnetic current meters (ECMs),

๑ Denotes content that is immediately available upon publication as open access.

Supplemental information related to this paper is available at the Journals Online website: https://doi.org/10.1175/JTECH-D-200115.s1.

Corresponding author: Pichaya Lertvilai, plertvil@ucsd.edu which utilize magnetic-induced electrical current by ions in seawater passing through a known magnetic field (Jones 1980), became popular in the 1950s and 1960s, but their popularity has been declining due to intrusive nature of the measurement (MacVicar et al. 2007). Drag force current meters are relatively inexpensive current sensors that measure drag force on known objects to calculate current velocity. These meters are widely used in ecological studies where instrument cost is the driving factor (e.g., Johansen 2014; Mach et al. 2011; Madin and Connolly 2006). Currently, acoustic current meters are the most popular way to measure currents where precise scientific data are desired. These current sensors measure either the Doppler shift or the travel time of transmitted acoustic signals that are backscattered from ambient particles, which allows the instruments to resolve flow velocity in two or three dimensions. There are two main popular variations of acoustic current meters, the acoustic Doppler current profiler (ADCP), which resolves a velocity profile of the water column, and the acoustic Doppler velocimeter (ADV), which measure a point velocity. Although these acoustic current sensors are widely used and can provide accurate velocity measurement (Lohrmann et al. 1994), these systems are often relatively expensive to use in high risk environments or swarm operations (Fuentes-Perez et al. 2018). They also have a large form factor and high energy consumption, which are not suitable for small platforms (Jaffe et al. 2017) or long-term deployment (Rudnick et al. 2004). As a result, development of new underwater current meters is still an active area of research.

A standard technology in velocity measurement uses optical systems to track movement of particles to measure currents, which are known as particle image velocimetry (PIV) and particle tracking velocimetry (PTV) (Prasad 2007; Raffel et al. 1989). In the case of aquatic systems, there are several systems that can perform in situ PIV or PTV measurements (e.g., Katija and Dabiri 2008; Liao et al. 2009; Tritico et al. 2007; 

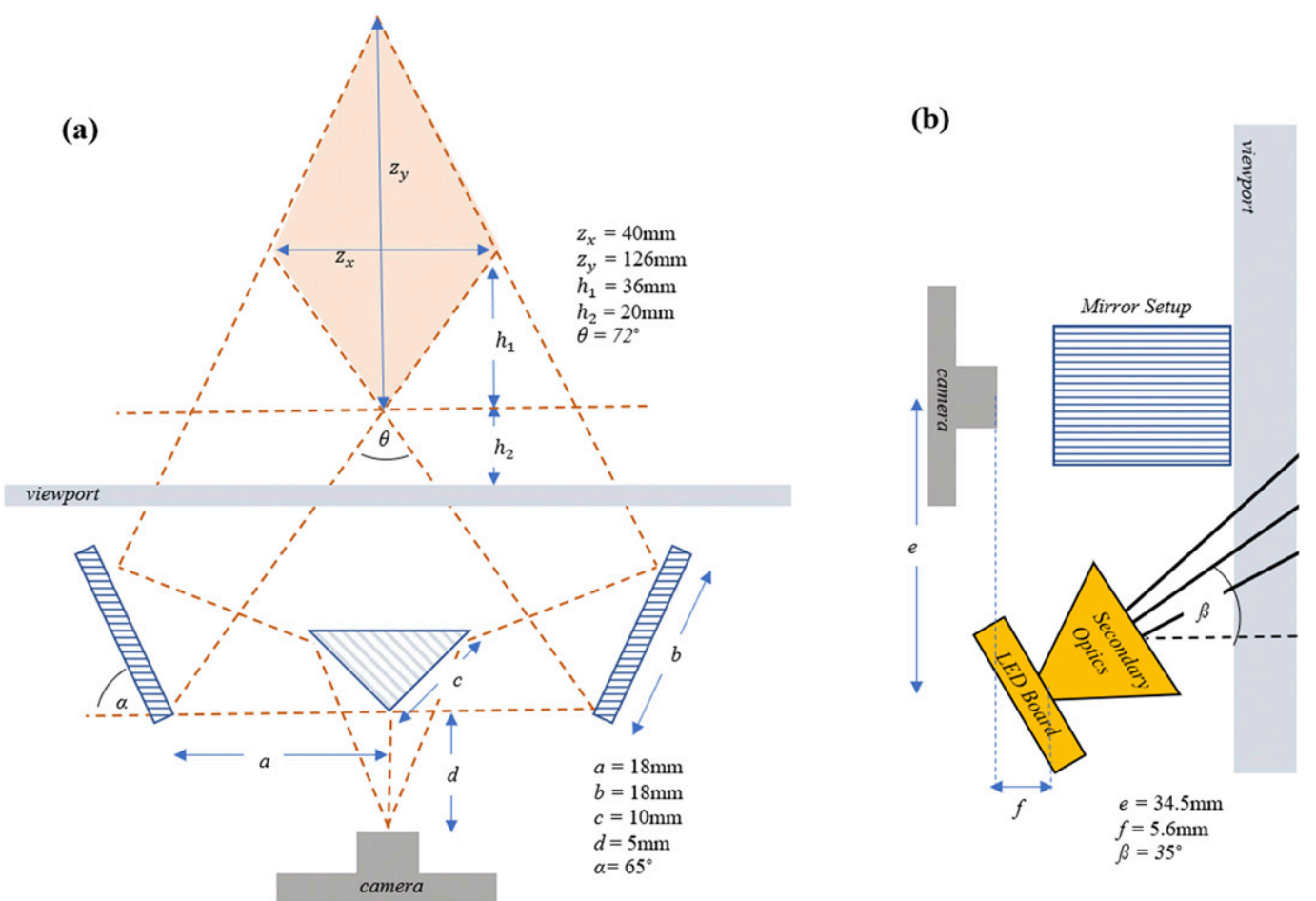

FIG. 1. Schematic of optics of the VIV. (a) The mirror setting that splits the view of the camera into two separate stereo views. (b) The position of the illumination LED with respect to the camera and the mirror setup. The secondary optics focuses the beam with full width at half maximum intensity of $14.3^{\circ}$. The mirror dimensions are from manufacturer's specifications, and the dimensions of the field of view are from calibration. The schematic is not drawn to scale.

Smith 2008). An in situ system for measuring turbulence using the PIV method has also been fabricated and tested (Steinbuck et al. 2010). There also are attempts to lower the complexity of PIV measurements by using LED illumination instead of a laser sheet, which resulted in more compact and more affordable PIV systems (Chételat et al. 2001; Hagsäter et al. 2008; Estevadeordal and Goss 2005). However, to date, these systems are not designed to be deployable in field environments.

Despite the utility of the in situ systems to resolve complex flow fields in multiple dimensions, there are many applications that only need a point measurement of velocity, similar to an ADV, and do not need the complexity of robust PIV or PTV techniques. Additionally, the emerging maker practice has enabled many electrical components and software packages that were traditionally costly or difficult to obtain, such as controllable cameras and single-board computers, to become widely accessible at low cost through collaborations between developers with open-source hardware and software (Tanenbaum et al. 2013). As a result, in this article, we explore the advantage of the maker practice to develop a less expensive and more compact system for point measurement of flow velocity in situ.

To achieve our goal, we constructed and calibrated a stereoscopic optical current meter based on a low-cost computer and camera system. A simplified particle image cross-correlation algorithm was then implemented to facilitate computation on the low-cost computer. The instrument was calibrated in a flume with controllable flow rate and then deployed in the field to capture in situ particle movements, which were then used to reconstruct the bulk flow velocity vector. The measurement was validated with an ADV, a standard velocity measurement technique. Here, we demonstrate that our system, which we refer to as the Video Velocimeter (VIV), is capable of estimating bulk flow velocity in situ with good agreement with an ADV using only ambient natural particles without additional particle seeding.

\section{Materials and methods}

\section{a. Instrument description}

The VIV utilizes a Raspberry Pi Camera V2 [Sony IMX219 sensor as detailed in Pagnutti et al. (2017)] with the stock lens as the imager and a Raspberry Pi single-board computer (Raspberry Pi Foundation, United Kingdom) as the main processing unit. To keep the hardware simple and to avoid problems of synchronization, a split field of view using one imaging sensor was employed as described in $\mathrm{Yu}$ and $\mathrm{Pan}$ (2016). The system consisted of a set of two planar mirrors and one right angle prism mirror to split the field of view into two views (Fig. 1). The mirrors and the prism were rated for 4-6 $\lambda$ flatness with protected aluminum coating (Edmunds Optics, United States). The size of the mirrors and the configuration $(a, b, c$, 

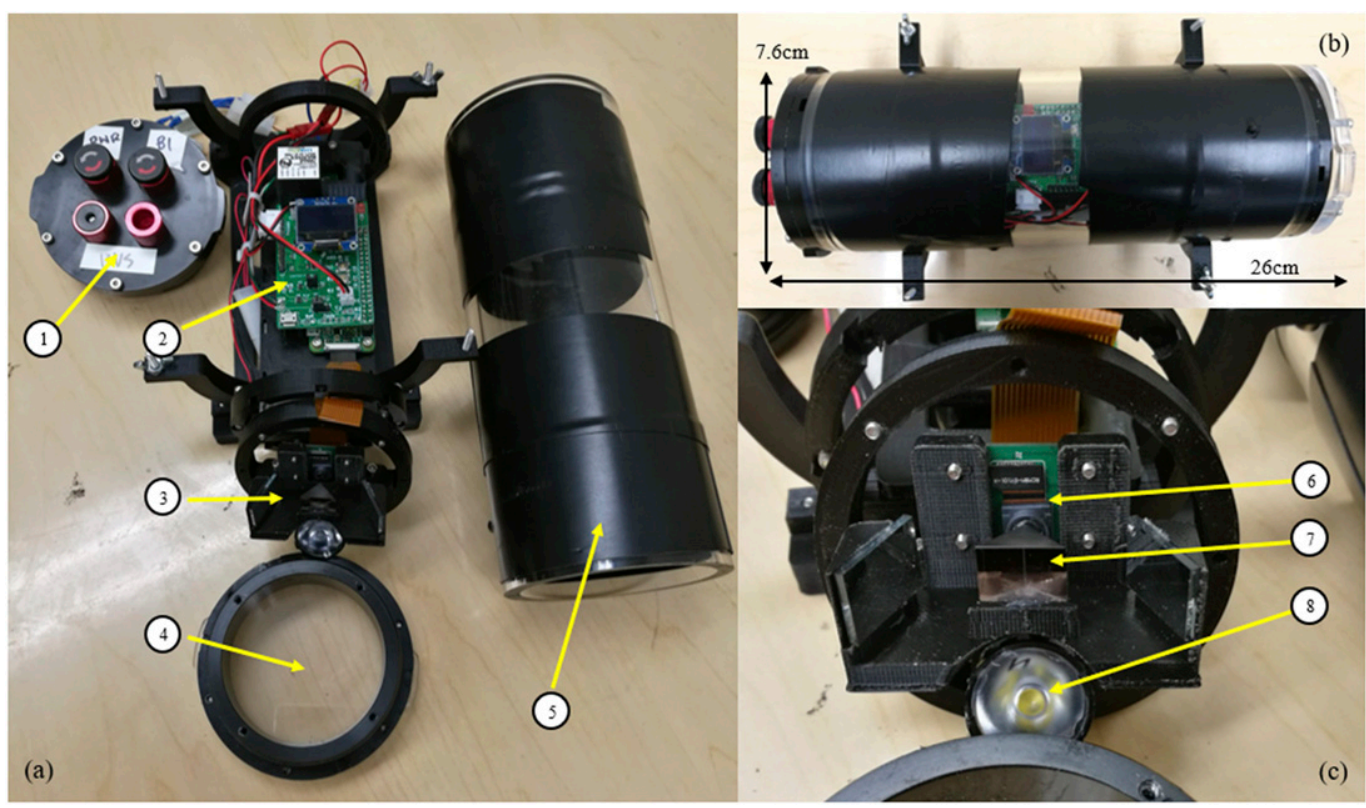

FIG. 2. The Video Velocimeter. (a) Overview of the entire instrument: 1) aluminum back plate with switches, pressure sensor and vent hole, 2) control board, 3) camera and optics, 4) acrylic view port, and 5) acrylic tube housing. (b) Assembled unit with dimensions. (c) Optical components: 6) Raspberry Pi camera, 7) mirror system, and 8) LED with lens.

and $\alpha$ ) were chosen to maximize the overlapping of the field of view and the illumination while allowing the setup to fit inside a commercially available waterproof housing. This method allowed inverting for a bulk $3 \mathrm{D}$ flow vector from the set of linearly independent views. The setup also reduced the computational overhead needed to control multiple cameras, which was significant for a small single-board computer.

To further minimize the complexity and the cost of the system, an LED illumination is chosen as the light source instead of a typical laser sheet illumination. LEDs produce incoherent light that suppresses speckles while costing much less than laser diodes and requiring simpler driver circuits (Chételat and Kim 2002). Due to space limitation inside the housing, a single CREE XT-E white LED was used as the light source. The LED utilized a Carclo frosted narrow spot lens as a secondary optics and is driven by a LuxDrive $500 \mathrm{~mA}$ BuckPuck DC LED driver (all illumination components were obtained from LEDsupply, United States).

For this ocean-going instrument, the Raspberry Pi Zero W model was chosen as the main computer because it was, at the time, the most power-efficient and the least expensive singleboard computer model. However, it should be noted that any Raspberry Pi model, including model $3 \mathrm{~A}+$ and $4 \mathrm{~B}$, could be interchanged for this instrument. The models with more computational power had the tradeoffs of costing more and consuming more power. In addition to the main computer, a custom-made control board was designed and fabricated that regulated power, communicated with a pressure sensor, and provided feedback through an OLED display (Fig. 2). The system was powered by a $24 \mathrm{Wh}$ lithium battery pack (Anker, Shenzhen, China), which allowed the instrument to run continuously for approximately $8 \mathrm{~h}$. The entire system was housed in an acrylic cylinder housing with an aluminum backplate and a flat acrylic viewport rated for $100 \mathrm{~m}$ depth (Bluerobotics, United States). The camera lens was adjusted so that the focal plane was $5 \mathrm{~cm}$ away from the front of the viewport. In 2019 dollars, the entire bill of materials for the instrument cost 480 U.S. dollars (Table 1).

It should be noted that the custom-made control board can be replaced with off-the-shelf boards that can perform power management for Raspberry Pi such as Sleepy Pi 2 (Spell Foundry, United Kingdom). Using off-the-shelf boards will help reduce lead time to fabricate the system. However, connections to other components, such as the LED driver and the environmental sensors, have to be manually wired and secured inside the housing. Caution should be exercised to make sure that connections are correct, and components are properly secured.

The VIV was programmed with Python code embedded on the Raspberry Pi using the default Raspberry Pi Camera

TABLE 1. Instrument cost breakdown materials. Note that the pressure sensor is not crucial for the function of the instrument as a velocity sensor but is included to obtain environmental information.

\begin{tabular}{lc}
\hline \multicolumn{1}{c}{ Items } & Cost (U.S. dollars) \\
\hline Housing & 130 \\
Pressure sensor & 90 \\
Optics & 135 \\
Camera & 30 \\
Computer & 10 \\
Control board & 60 \\
Battery & 25 \\
Total & 480 \\
\hline
\end{tabular}



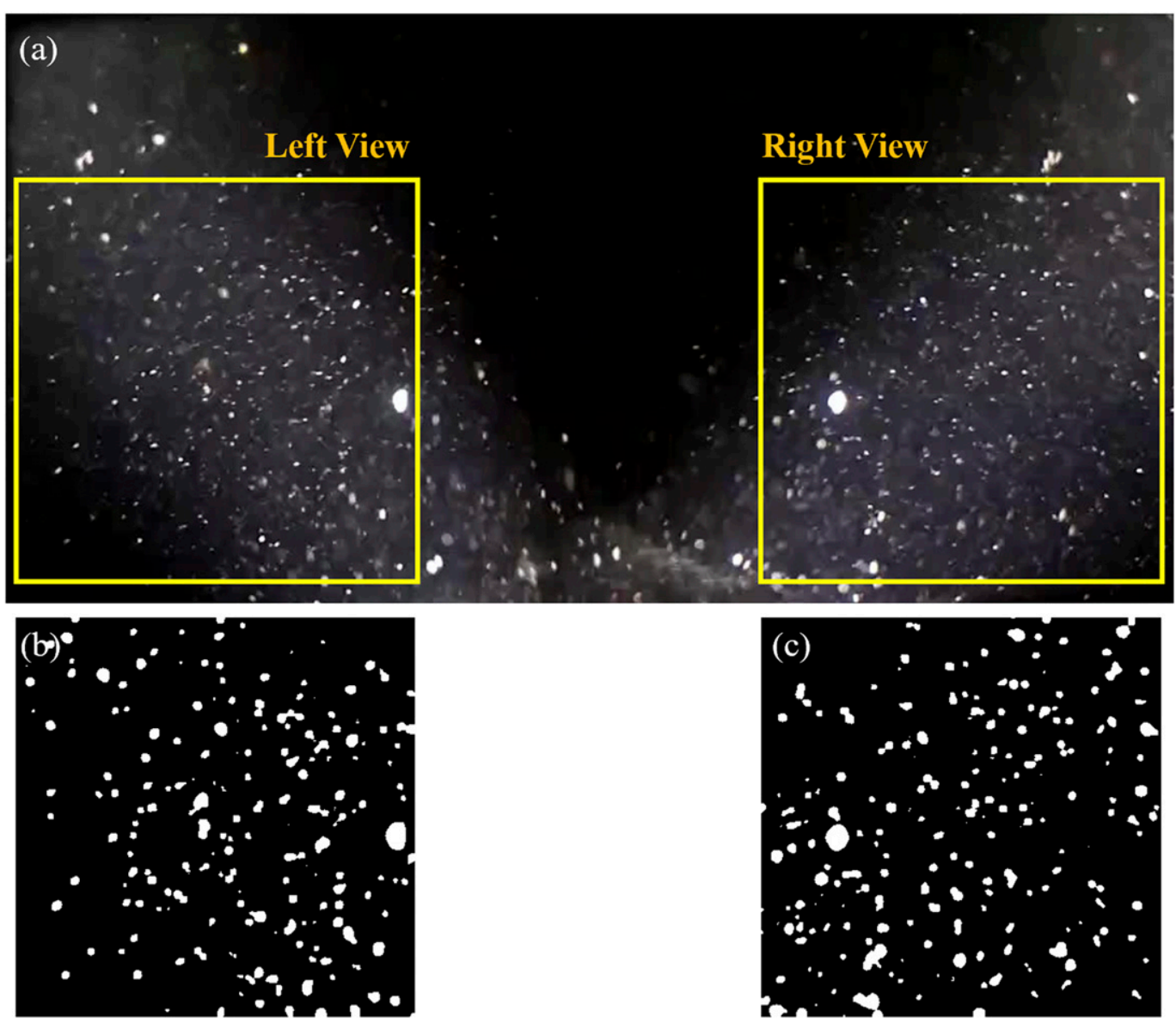

FIG. 3. An example image of ambient particles recorded by the VIV. (a) The full field of view of the camera, which is split into two linearly independent views. The yellow boxes indicate the regions that are extracted to perform velocity measurement. (b),(c) The images of particles used in velocity measurements of the simplified correlation method. The images are preprocessed with adaptive thresholding to enhance particles and to equalize their intensity within particles.

package provided by the Raspberry Pi Foundation. The camera software allowed the computer to adjust setting of the camera and also provided a flash synchronization feature. This feature enabled the LED to be hardware triggered by a control pin to synchronize the flash with the frame acquisition. This flash synchronization allowed the power consumption of the LED to be half of what it would be if the LED were constantly on.

This implementation, together with the volume illumination, allowed the entire setup to fit inside a small cylindrical housing of $7.6 \mathrm{~cm}$ diameter $\times 26 \mathrm{~cm}$ long. The trade-off was that the usable field of view (FOV) of each stereo view was reduced to half of the original camera FOV. Given the mirror setup, the usable FOV was also smaller than the sensor of the Raspberry Pi Camera V2, so each view was obtained by extracting a $400 \times 400$ pixel region, which was then used to estimate velocity (Fig. 3a).

\section{b. Velocity reconstruction with simplified correlation}

The LED illumination in the VIV resulted in volume illumination. In a typical microPIV system, the imaged particles are constrained by the narrow depth of field of the imaging systems (Bourdon et al. 2003; Olsen and Adrian 2000). However, the low-cost Raspberry Pi Camera provided a much broader depth of field, in the centimeter range, when the focal plane was set to $5 \mathrm{~cm}$ distance from the front of the viewport of the VIV. With only two views provided by the mirror setup and the low computing power of the Raspberry Pi computer, it was not feasible to accurately implement velocity field reconstruction. However, in this study, we only seek to compute the averaged velocity of all particles in the field of view in contrast to the detailed flow field. As a result, we will only implement a simplified correlation algorithm as formulated below.

First, even though the optical setup was designed such that the LED illuminates all particles in the overlapping FOV of the two views, the nonuniform illumination of the LED causes particles in the middle of the FOV to be brighter than those on the edges (Fig. 3a). To correct for this nonuniformity, raw images from the camera were preprocessed by Wellner's adaptive thresholding method (Wellner 1993) to create binary images of particles (Figs. 3b,c). This process allows all particles 
that are brighter than the immediate surrounding background to be detected regardless of the absolute intensity of both background and particles. This process also detects particles in the overlapping FOV that are out of focus in each view and treat them the same as in-focus particles. With this processing step, the raw images become binary images such that all detected particles have the same uniform intensity and are approximately spherical.

With a large depth of field, the location of the peak of the correlation function is the weighted sum of the individual correlation functions at each range $z$ from the imager (Bourdon et al. 2003):

$$
C_{v}(x, y)=\alpha \sum_{z=z_{\min }}^{z_{\max }} c(x, y, z)
$$

where $C_{v}$ is the overall correlation of the entire volume, $c(x, y, z)$ is the individual correlation at range $z, \alpha$ is a proportional constant, and $z_{\min }$ and $z_{\max }$ are the boundaries in range of the illuminated particle field. Typically, the volumetric correlation is accomplished by decomposing the image with regards to point spread function of seed particles with known size (Fouras et al. 2009). However, the VIV is intended to use natural particles, so the particle sizes cannot be known a priori.

Opportunistically, medium-scale oceanographic currents, such as internal waves and wind-driven waves, are often periodic and unidirectional in short periods of measurement time (Garwood et al. 2020; Johansen 2014). As a result, we can make simplifying assumptions that all particles in the illuminating volume are moving at the same velocity and are uniformly distributed in the volume. We further assume that particle size distribution is constant within the volume such that the average size of the imaged particles at every range is the same. Since we use binary images from the adaptive thresholding step, we can model these particles as approximately spherical with a projection of uniform circles of average radius $r$. The correlation peak of each depth would be located at $-f \Delta(X, Y) / z$, where $f$ is the focal length of the imager and $\Delta(X, Y)$ is the particle displacement. These assumptions simplify the correlation function at each range to

$$
c(x, y, z)=\alpha I_{0}(z)\left[1-\frac{\left|(x, y)-\frac{f(\Delta X, \Delta Y)}{z}\right|}{r}\right],
$$

when $f(\Delta X, \Delta Y) / z-r \leq(x, y) \leq f(\Delta X, \Delta Y) / z+r$, and $c(x$, $y, z)=0$ otherwise. The intensity of particles $I_{0}(z)$ is proportional to $1 / z^{2}$ (Olsen and Adrian 2000). We can then substitute (2) into (1) and take the integral form of the Riemann sum as

$$
C_{v}(x, y)=\beta \int_{z_{\min }}^{z_{\max }} \frac{1}{z^{2}}\left[1-\frac{\left|(x, y)-\frac{f(\Delta X, \Delta Y)}{z}\right|}{r}\right] d z,
$$

where $\beta$ is a constant. Solving the integral yields the peak location at

$$
(\hat{x}, \hat{y})=\frac{f}{\left(\frac{z_{\min }^{3}+z_{\max }^{3}}{2}\right)^{1 / 3}}(\Delta X, \Delta Y)=M(\Delta X, \Delta Y) .
$$

As a result, the measured displacement $(\hat{x}, \hat{y})$ from this simplifying correlation is proportional to the real displacement $(\Delta X, \Delta Y)$.

The proportional constant $M$ only depends on the system parameters, so it can be obtained from laboratory calibration. However, even though this proportional constant does not depend on the particle size distribution, the size distribution still affects the shape of the cross correlation as seen in (3). In real implementation, there will be out-of-focus particles and occasional large particles that can cause the cross-correlation map to be noisy. This noise can reduce the accuracy of the peak detection in the algorithm, resulting in a less reliable velocity estimation. As a result, it is important to track the signal-tonoise ratio (SNR) of the correlation, which represents the measurement uncertainty in velocity estimation. In this study, we will use the primary peak ratio method (Xue et al. 2014),

$$
\mathrm{SNR}=\frac{P_{1}}{P_{2}}
$$

where $P_{1}$ is the magnitude of the primary peak of the crosscorrelation map, and $P_{2}$ is the magnitude of the secondtallest peak.

For the VIV, $400 \times 400$ pixels regions from both views of the camera are processed with adaptive thresholding method to generate binary images. These binary images are then cross correlated to compute the projected velocities from left view and right view, designated as $v_{1}=\left(v_{x 1}, v_{y 1}\right)$ and $v_{2}=\left(v_{x 2}, v_{y 2}\right)$, respectively. We implemented this cross correlation and peak detection using the OpenPIV Python package (Liberzon et al. 2016). Next, the apparent velocities from both views, $V_{1}$ and $V_{2}$, are obtained by multiplying the projected velocities with the multiplicative constant $M$ obtained from laboratory calibration. Finally, both $V_{1}$ and $V_{2}$ are used to perform stereo reconstruction with the extrinsic parameters of the two views to compute the true velocity in three dimensions $V=V_{x}, V_{y}, V_{z}$ (Prasad 2000). This process is outlined in Fig. 4.

\section{c. Laboratory validation}

All optical calibrations of the instrument were done by submersing the instrument in a glass tank filled with filtered seawater and covered by black electrical tape on the inside to reduce reflection. The stereo view was calibrated with a checkerboard pattern with $6 \times 4$ inner corners using the photogrammetric algorithm (Zhang 1999) provided by OpenCV package. The angle between the two views was thereby calibrated to be $72^{\circ}$ ( $\theta$ in Fig. 1). The overlapping FOV of the two views are obtained by tracing the edge of the FOV of each stereo view on a paper; the dimensions are shown in Fig. 1.

To optimize the exposure and gain of the VIV an experiment was formulated that was designed to simulate a distribution of natural particles that would be encountered in the field, however, with known reflectors of known sizes. Here, we used polystyrene beads (Polyscience Inc., United States) of sizes $5-90 \mu \mathrm{m}$ diameter at concentrations of $8-150$ beads per milliliter. The camera recorded video at $65 \mathrm{fps}$ with $1280 \times 720$ resolution. The exposure and gain were optimized by using the particle detection algorithm based on using the adaptive thresholding method to discern whether the camera could 


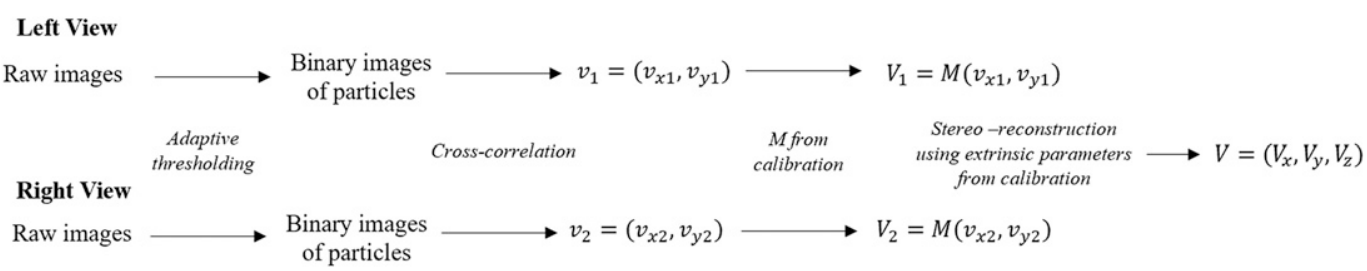

FIG. 4. Outline of the algorithm used in the VIV to reconstruct the 3D flow velocity from the images obtained from both views of the system. Here lowercase $v$ indicates projected velocities, and uppercase $V$ indicates real velocities.

detect the beads. Based on these experiments, it was concluded that a camera exposure of $2.5 \mathrm{~ms}$ with ISO320 was optimal. With this setting, the instrument could reliably detect polystyrene beads of $50 \mu \mathrm{m}$ diameter or larger.

To test the system in uniform flow, an experimental Vogel flume (Lacoursière and Craig 1990) was constructed from clear acrylic sheets with the dimension $120 \mathrm{~cm} \times 15 \mathrm{~cm} \times 20 \mathrm{~cm}$. The camera was attached to the outside wall such that the flow was parallel to the horizontal axis of the image, and it was positioned $60 \mathrm{~cm}$ away from the flume inlet and $8 \mathrm{~cm}$ above the bottom. The flume was filled with unfiltered natural seawater collected from the end of Scripps Pier, La Jolla, California.

Furthermore, in order to compare the method with a more traditional sensor as well as getting a correct estimate of velocity, a $10 \mathrm{MHz}$ Sontek ADV was used concurrently to measure the 3D flow speed. The sampling volume of the ADV was located $8 \mathrm{~cm}$ above the bottom and as close to the imaging volume as possible without obstructing the LED illumination or disturbing the nature of the unidirectional flow. Flow speed inside the flume was controlled by a recirculating pump and was varied from $5 \mathrm{~cm} \mathrm{~s}^{-1}$ to a limit of $30 \mathrm{~cm} \mathrm{~s}^{-1}$. At each flow speed, the ADV was used to measure the velocity for $60 \mathrm{~s}$, and the average speed over this period was used to compute the displacement in the object plane $\Delta X$ of the particles by multiplying the speed by the time interval between consecutive frames captured by the VIV. The VIV took a sequence of 30 images, and the simplified correlation method was used to reconstruct velocity. The ensemble average over the 30 frames was then calculated to validate the linearity in (4) in both the uniform flow and more turbulent conditions. The two-component velocities from each view of the VIV were then used to triangulate the three-dimensional flow. Results were then compared to the ADV measurements.

\section{d. Field experiment}

For field measurements, the VIV was mounted on a speed rail rig with its cylindrical axis parallel to the seafloor. An Argonaut ADV and an FT2ADV probe (Sontek, United States) were also mounted on the same rig (Fig. 5). However, only the data from the FT2ADV were used in this comparative experiment. The systems were configured so that the sampling volume of the ADV was inside the sampling volume of the VIV (Fig. 5b). The FT2ADV sampling frequency was set to $2 \mathrm{~Hz}$. The rig was deployed by divers approximately $75 \mathrm{~m}$ west of Scripps Pier, La Jolla, at $13 \mathrm{~m}$ depth with the sampling volume located approximately $1 \mathrm{~m}$ above the seafloor. The instruments were positioned such that $V_{x}$ measures alongshore velocity, $V_{y}$ vertical velocity and $V_{z}$ across shore velocity (Fig. 5b). The instruments were set to continuously collect data after positioning.
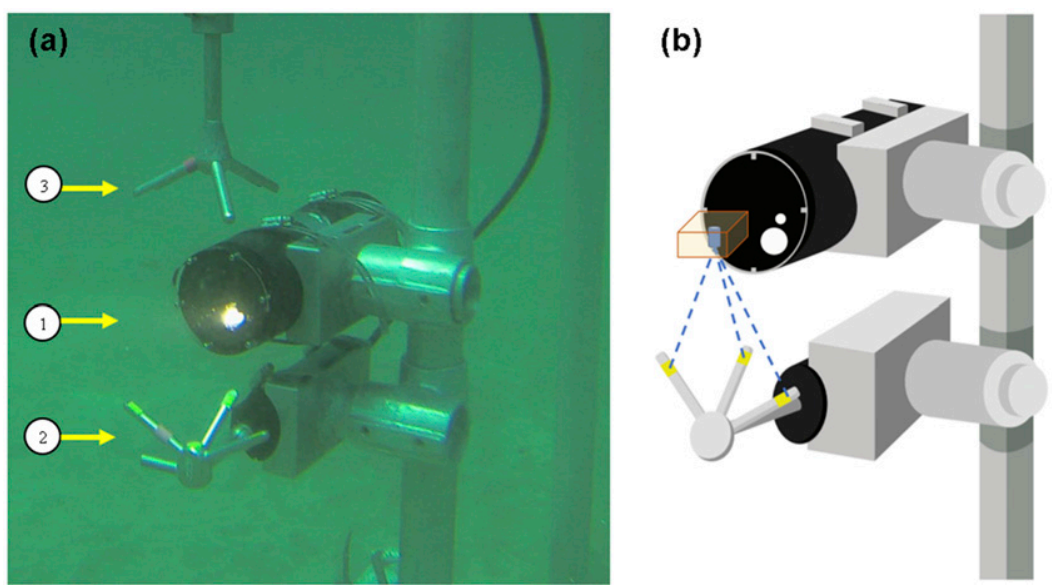
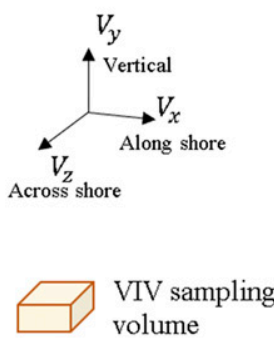

ADV sampling volume

FIG. 5. (a) Field deployment of 1) the VIV with 2) an Argonaut ADV and 3) an FT2ADV. The site is 75 m west of Scripps Pier, La Jolla, at approximately $13 \mathrm{~m}$ depth. The VIV is positioned $1 \mathrm{~m}$ above the seafloor. (b) Schematic of the deployment configuration showing the sampling volume of the ADV inside the sampling volume of the VIV and the flow direction with respect to the shore. 

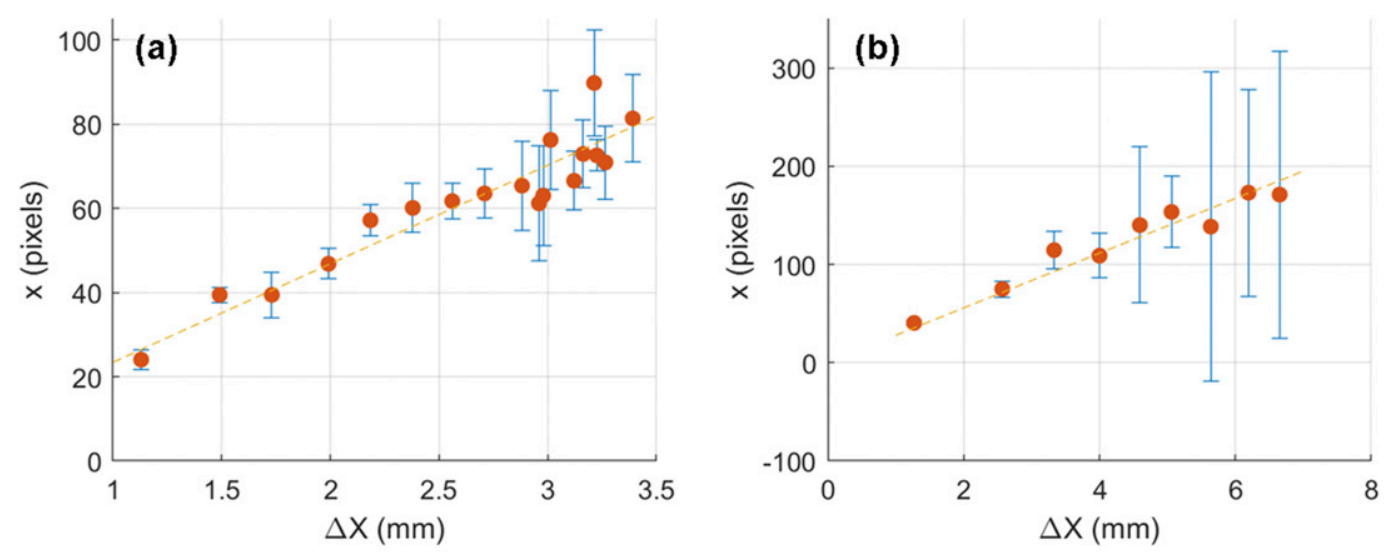

(c)

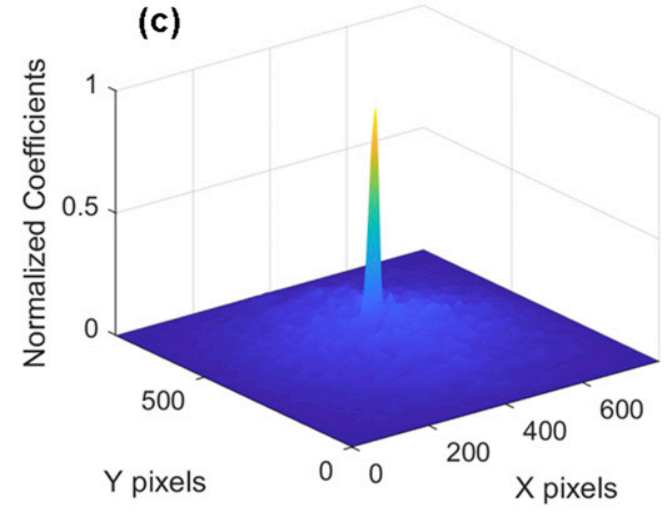

(d)

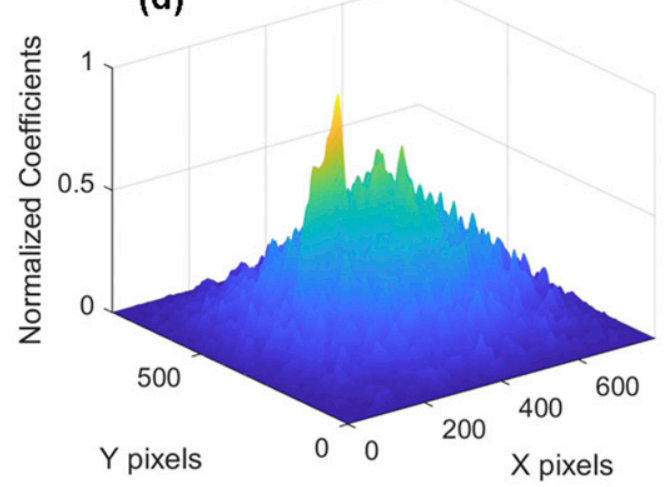

FIG. 6. Laboratory validation of linearity between the averaged displacement measured by the ADV $\Delta X$ and the averaged projected displacement $X$ evaluated by the simplified correlation method (a) in slow unidirectional flow conditions and (b) in faster and more turbulent flow. (c) The cross-correlation map in a slow flow condition at $\Delta X=1.5 \mathrm{~mm}$ shows a very distinct and narrow peak. (d) The cross-correlation map in more turbulent condition at $\Delta X=6.5 \mathrm{~mm}$ shows a noisy map with less distinct peak.

The video record from the VIV was analyzed on each of the two fields of view of the camera system to obtain an estimate of the velocities in the camera coordinate system. The pair of vector velocity values, in the coordinate of the camera fields of view, were then used to estimate a velocity vector. Next, the result of nine velocity values that were obtained from the 10 pairs of consecutive frames were averaged to achieve one ensemble velocity vector (Meinhart et al. 2000). Given the systems frame rate of $65 \mathrm{fps}$ and the fact that 10 frames were used to obtain each velocity, the time interval between the successive velocity estimates was $154 \mathrm{~ms}$. As the FT2ADV and the VIV were not hardware synchronized, data from the two instruments were synchronized by cross correlating the time series of both instruments to find the time offset. The time offset was then used to realign the velocity measurement from the VIV and the FT2ADV. To compare the velocity measurement at a given time, the velocity data from the FT2ADV were interpolated to match the sampling frequency of the VIV.

The SNR values calculated by the primary peak ratio method as described in section $2 \mathrm{~b}$ were also recorded to determine the reliability of each velocity estimate. The number of particles detected in each frame and their size range after the adaptive thresholding step were also reported.

\section{e. Real-time processing}

In many remote applications, such as long-term mooring and autonomous platforms, the VIV might be deployed for a long period of time, and the recorded videos might not be readily accessible to perform the velocity measurement until after the deployment. As a result, it is desirable for the VIV to perform real-time processing with the internal computer. The real-time velocity measurement would allow the VIV to use significantly less data storage and also allow the velocity measurement to be sent remotely as a small data packet to inform a remote vehicle about its relative velocity.

To explore the ability of the VIV to perform real-time velocity measurement, data from the field deployment were used to benchmark the processing speed of several candidate internal computers. For this study, three models of Raspberry Pi: model Zero W, 3A+ and 4B (2 GB RAM) were evaluated. The power consumption of the computers during the analysis was also recorded by measuring the current draw of the computers. 

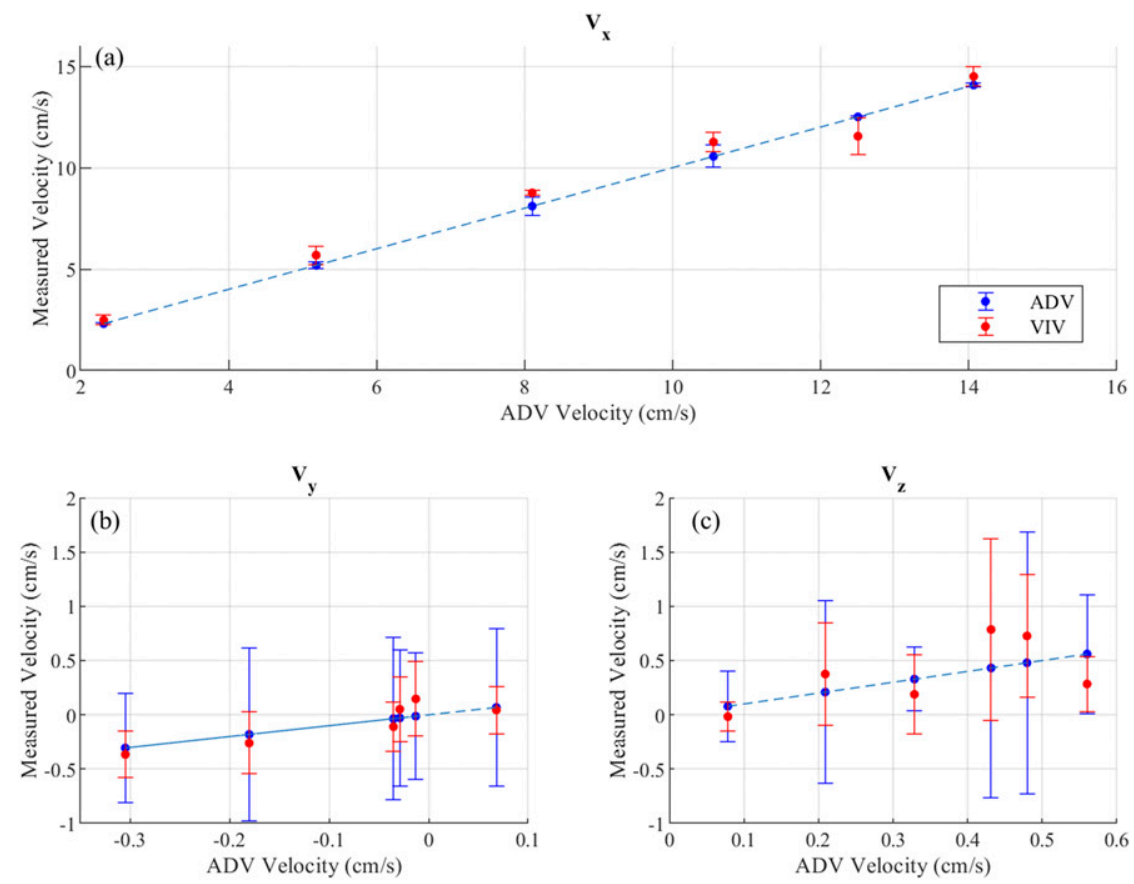

FIG. 7. The flow velocity as measured by the FT2ADV and the VIV. Each data point of the $\mathrm{ADV}$ is the average velocity value over $60 \mathrm{~s}$, and the error bar indicates the standard deviation. Each data point of the VIV is the ensemble average over 30 consecutive frames, and the error bar indicate the standard deviation of the velocity within the ensemble average.

Additionally, a Jetson Nano developer kit (Nvidia), another low-cost single-board computer, was used because it represents a family of single-board computer with a more powerful graphical processing unit that enables accelerated image processing. It should be noted that Jetson Nano consumes more power than Raspberry Pi computers and also has a larger footprint that does not fit inside the current VIV housing. As such, the result from the Jetson Nano is used to demonstrate the potential for future improvement of the VIV. The detail of testing conditions can be found in the online supplementary material section S1.

\section{Results}

\section{a. Laboratory validation}

In the laboratory experiment, at slower flow speed, the flow in the flume exhibits minimal fluctuation as measured by the ADV (Fig. S1). The results from these slower and unidirectional flows (Fig. 6a) indicate linearity between the displacement in the object plane $(\Delta X, \Delta Y)$ and the calculated displacement in the image plane $(\hat{x}, \hat{y})$ as predicted by (4). The cross-correlation map of two consecutive frames (Fig. 6c) shows a very distinct and narrow peak, which indicates high SNR of the velocity measurement.

However, as the flow became faster and more turbulent as shown by the ADV measurement (Fig. S1b), the crosscorrelation map between two frames becomes very noisy, and the primary peak is less distinct from the rest of the map (Fig. 6d). Even though the velocity estimation of the ensemble average over 30 frames still produces linear relationship, the high standard deviations and noisy correlation maps indicate that the estimations in this regime are unreliable (Fig. 6b). This result emphasizes the limitation of the simplified correlation method that requires uniform flow.

The measurements using the stereo mirror setup in the slow flow regime are shown in Fig. 7 to compare the velocity measurement of the ADV to that of the VIV. As measured by the ADV over $60 \mathrm{~s}$, the unidirectional flow in the flume $\left(V_{x}\right)$ was varied from 2 to $14 \mathrm{~cm} \mathrm{~s}^{-1}$. The velocity in the other two directions $\left(V_{y}\right.$ and $\left.V_{z}\right)$ fluctuates around $0 \mathrm{~cm} \mathrm{~s}^{-1}$. The comparison between the two instruments shows that the velocity measured by the VIV falls within the value measured by the ADV within one standard deviation of each instrument, indicating good agreement between the two instruments.

\section{b. Field experiment}

Figures 8 and 9 show the comparison of the velocity measured by the VIV and the FT2ADV over 150 s from the field deployment. The raw data of the VIV are color-coded by the SNR values as calculated by the primary peak ratio method. As shown, the raw velocity estimation of the VIV has good agreement with the ADV in $V_{x}$ and $V_{y}$ when the SNR values are above $10 \mathrm{~dB}$, but the estimation fluctuates rapidly and becomes less reliable as the SNR drops below 10dB. However, the VIV consistently underestimates the velocity in the $z$ direction regardless of the SNR.

For $V_{x}$ and $V_{y}$, a velocity estimation with SNR $>10 \mathrm{~dB}$ has a cross-correlation map similar to Fig. 10b where the narrow primary peak can be reliably detected. If smaller interrogation 


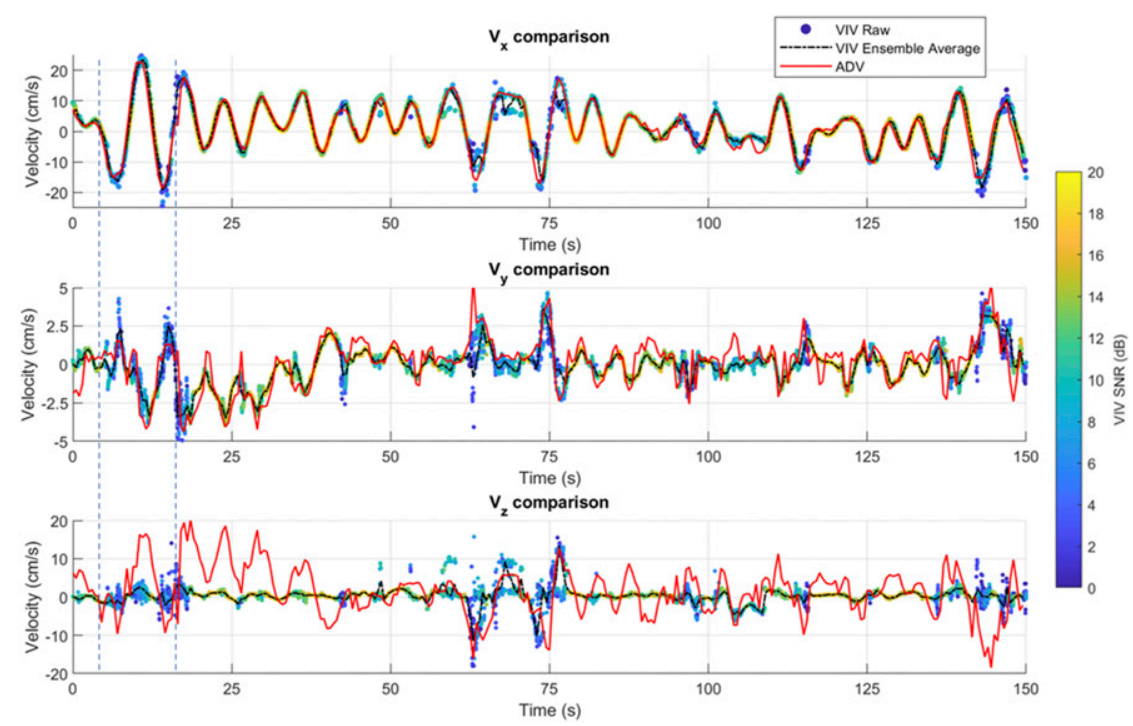

FIG. 8. The time series comparison of the data from the VIV and from the FT2ADV probe the field deployment. VIV raw is the instantaneous velocity estimate from the VIV between every consecutive frame recorded at $65 \mathrm{fps}$. The color of VIV raw represents the signal-to-noise ratio (SNR) calculated from the primary peak ratio of cross-correlation maps. VIV ensemble average is the ensemble average of VIV raw over 10 velocity estimates. The two vertical dashed lines indicate the time points at 4.8 and $16.4 \mathrm{~s}$, which are reviewed in detail in Fig. 10.

windows of $100 \times 100$ pixels with $50 \%$ overlap are used to perform cross correlation, the result in Fig. 10c shows that particles in each region are moving in the same general direction, validating the uniform flow assumptions of the simplified correlation method. However, the SNR of each small interrogation window can be different from the overall cross correlation. In particular, the regions with out-of-focus particles, which corresponds to larger binary mask, such as regions on the left edge of Fig. 10a, the cross-correlation maps can be noisy and yields lower SNR values. On the other hand, the correlation map of a velocity estimation with SNR $<10 \mathrm{~dB}$ (Figs. 10d-e) shows a noisy map with a broader primary peak. The correlation of smaller integration windows show that particles are moving in different directions throughout the field of view of the camera (Fig. 10d). This behavior violates the assumptions of the simplified correlation method, rendering the data point to become less reliable. In this periodic flow, the SNR of the VIV is typically high when the main flow is accelerating, but the SNR drops when the acceleration slows down to change direction at peaks and troughs of the velocity oscillation.

Even though the velocity estimation with low SNR values causes the raw data to fluctuate, this issue can be alleviated with the ensemble average of 10 consecutive frames. This timeaveraged method improves the root-mean-square errors of $V_{x}$ and $V_{y}$ by $42 \%$ and $47 \%$, respectively, as shown in Fig. 9. The ensemble average data show a good agreement in $V_{x}$ and $V_{y}$ with low root-mean-squared errors of 1.43 and $0.5 \mathrm{~cm} \mathrm{~s}^{-1}$ within the velocity range of 20 and $5 \mathrm{~cm} \mathrm{~s}^{-1}$, respectively. As shown, the VIV can measure the flow speed up to $20 \mathrm{~cm} \mathrm{~s}^{-1}$ with a good agreement with the ADV.

\section{c. Real-time performance}

Table 2 summarizes the results from all tested single-board computers. The Raspberry Pi Zero W, which was used in the presented ocean-going VIV, has a relatively slow processing rate of five seconds per velocity estimate. The more powerful Raspberry Pi models $3 \mathrm{~A}+$ and $4 \mathrm{~B}$ can approximately estimate one velocity per second but consumes more power, which can limit the amount of time the instrument can be deployed autonomously with the same amount of battery power. The Jetson Nano can perform at a much faster rate of 3.76 estimations per second, but the power consumption of this powerful processing unit is triple the rate of the Raspberry Pi Zero.

\section{Discussion}

\section{a. Velocity estimation}

The results from the laboratory and the field experiment demonstrate that the VIV and its processing algorithm can estimate the flow parallel to the face of the viewport well when the flow is approximately uniform in the sampling volume. When the flow becomes more turbulent, an individual velocity estimation is less reliable with low SNR value, but the unreliability can be alleviated by performing ensemble average over a short period of time. However, the VIV consistently underestimates the axial velocity component that is perpendicular to the viewport.

This discrepancy in $V_{z}$ is a result of larger sampling volume of the VIV compared to the ADV. The FT2ADV has a small cylindrical sampling volume with $6 \mathrm{~mm}$ diameter and $9 \mathrm{~mm}$ height, while the VIV has a sampling of volume of 

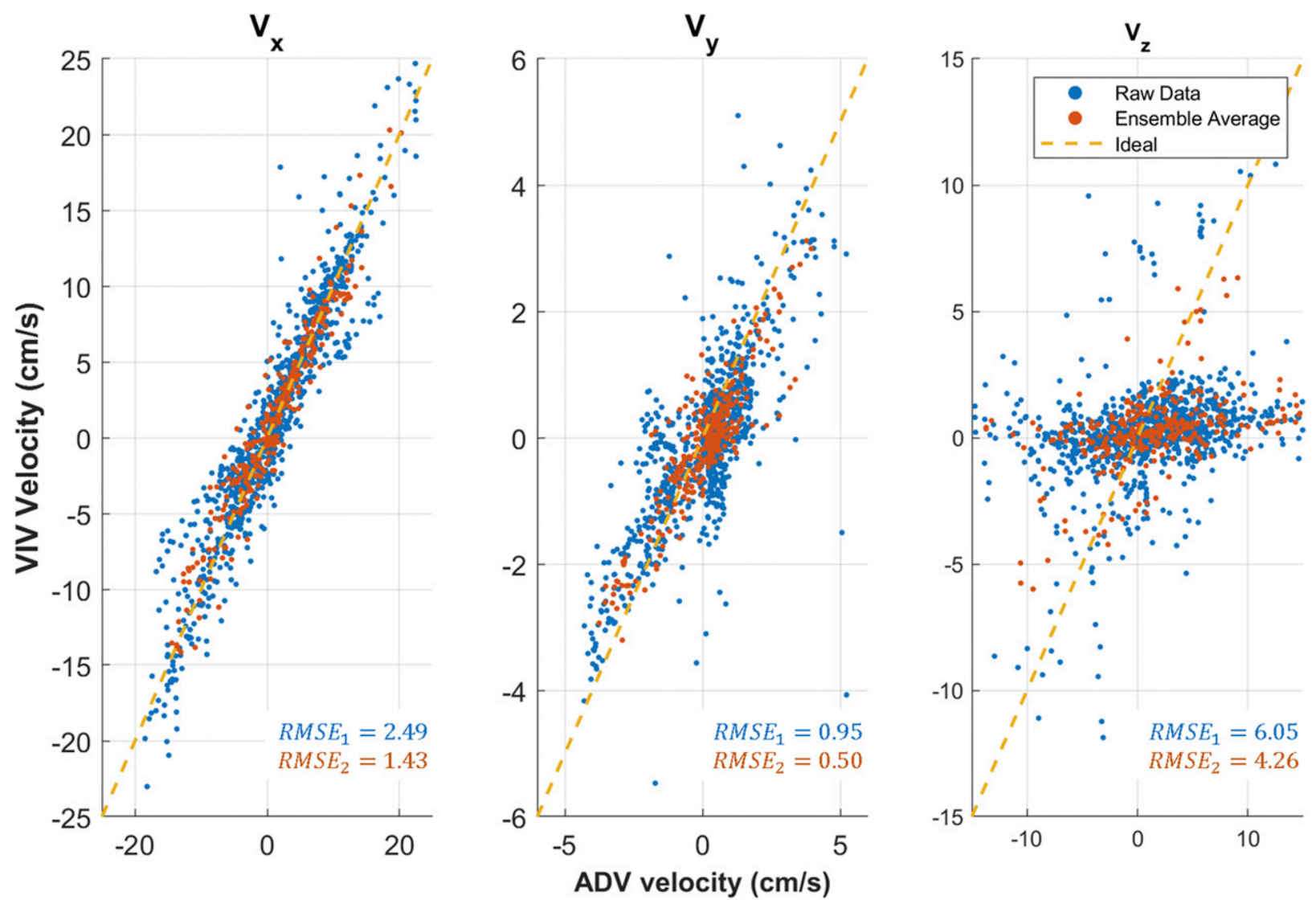

FIG. 9. The scattered plots of the data from the raw VIV measurement (blue) and from the ensemble average (red) compared to the

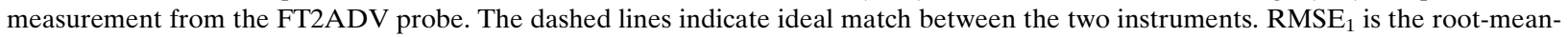
squared error calculated from the raw VIV measurement, while $\mathrm{RMSE}_{2}$ is calculated from the ensemble average.

approximately $20 \mathrm{~mm} \times 20 \mathrm{~mm} \times 20 \mathrm{~mm}$. The experiment was setup such that the sampling volume of the ADV was in the middle of the sampling volume of the VIV. When averaging over the entire sampling volume, the VIV sampled more particles that were closer to the viewport of the instrument. The speed of these particles in $z$ direction was reduced due to the blocking from the cylindrical, which is $7.6 \mathrm{~cm}$ in diameter. As a result, when averaging over the entire sampling volume, the slower speed of these closer particles resulted in underestimation of the $V_{z}$ component.

This limitation in the axial flow estimation is circumstantial based on the constraints of the housing diameter. If all three velocity components are needed, the VIV can be scaled up to larger housing that allows the mirrors to be tilted more and moves the overlapping field of view farther away. The illumination can also be moved outside of the housing to accommodate the new configuration. This adjustment would allow more accurate 3D studies with the trade-off of the compact size of the instrument.

With the current iteration of the VIV, the instrument is mainly suitable for estimating nonturbulent slow flow and is not a perfect replacement of an ADV, which can be used in more complex flow regimes. We note, that in order to address the issue of reliability we caution users to carefully observe the
SNR of each velocity estimation, and when the SNR values become low, the system's estimate of the mean velocity is likely to be unreliable. Additionally, the VIV is limited to estimating flow parallel to the face of the viewport, so the orientation of the instrument is critical in an actual flow study. Accordingly, the instrument should be oriented such that the main flow is parallel to the face of the viewport, so the main velocity components can be estimated.

Despite this limitation, the VIV has potential for many practical uses. Many ecological studies do not need to resolve all three velocity components to understand the ecological implications of the ocean current (Madin and Connolly 2006; Mach et al. 2011; Johansen 2014). Moreover, the low cost of the system permits increased feasibility so that multiple units could be used to cover a larger spatial area in a single deployment. The deployment of multiple units will be particularly effective in recording and understanding the flow over a complex benthic structure with relatively low flow rate, such as coral reefs. Presently, this type of study is currently being done with multiple ADVs (Hench and Rosman 2013; Huang et al. 2012) or ADCPs (Gaylord et al. 2007; Rogers et al. 2018). Since the spatial variability in flow is of importance, increased spatial sampling density for the same price is definitely of advantage. 
(a)

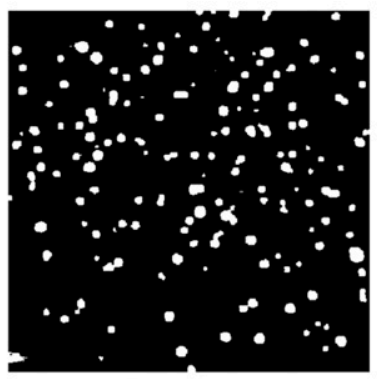

(d)

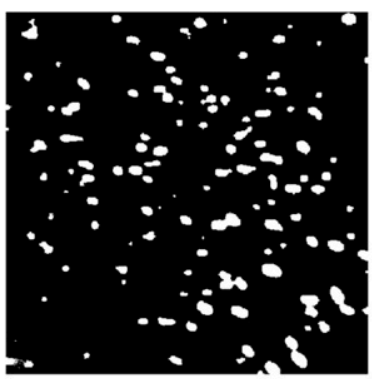

(b)

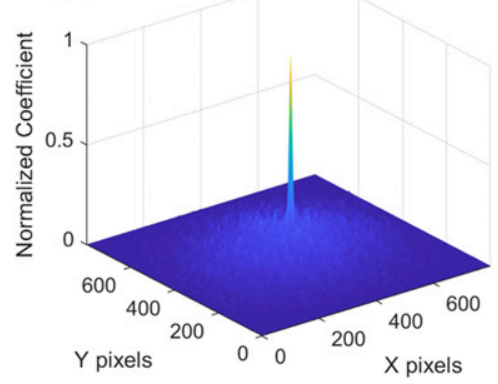

(e)

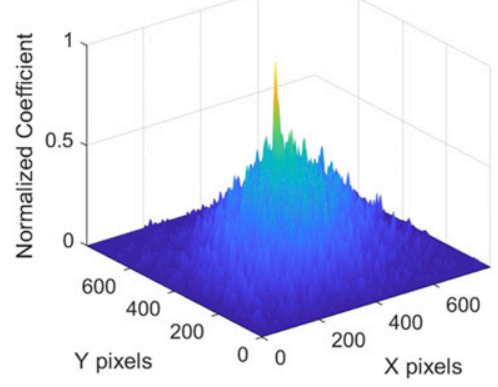

(c)

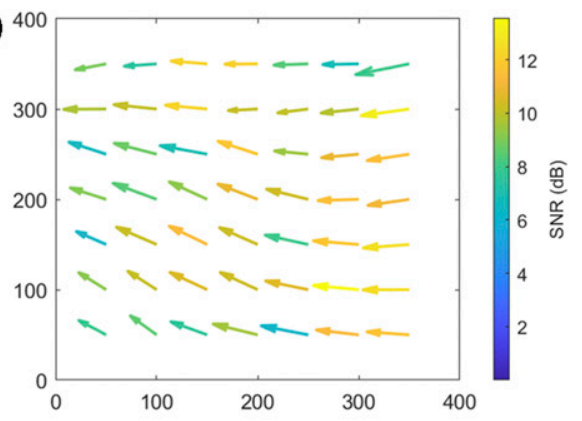

(f)

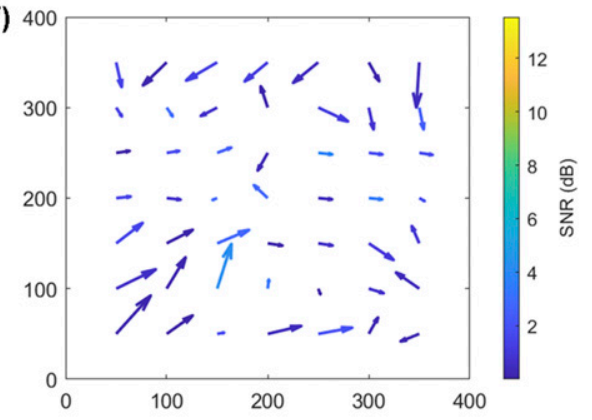

FIG. 10. Examples of cross-correlation maps from two velocity estimates with high and low SNR from the field deployment. The time points are marked by dashed lines in Fig. 8. The cross-correlation maps are obtained from cross correlating the entire $400 \times 400$ pixel fields of view of the VIV. At $t=4.8 \mathrm{~s}$, (a) the binary image of particles in the region of interest corresponds to (b) a velocity estimate with a high SNR of $15 \mathrm{~dB}$. (c) The corresponding velocity field, obtained from smaller $100 \times 100$ pixel interrogation windows with $50 \%$ overlap, shows flow of particles in similar direction. The color of the arrows represents the SNR field of each smaller interrogation windows. At $t=16.4 \mathrm{~s}$, (d) the binary image of particles corresponds to (e) a velocity estimate with a low SNR of $5 \mathrm{~dB}$. (f) The corresponding velocity field shows particles moving in different directions. Both correlation maps yield similar velocity estimate in the main flow of approximately $1.5 \mathrm{~cm} \mathrm{~s}^{-1}$.

\section{b. Ambient particles}

During the field deployment, the particles that were detected by the adaptive thresholding method in each frame, have the mean equivalent radii between 4 and 6 pixels (Fig. 11a) with overall average standard deviation of 2.8 pixels. This narrow range of particle size within each frame and also between frames indicates that the assumption of uniform size distribution was valid in this deployment. The instrument also experiences particle counts in the range of 5-20 particles per milliliter (Fig. 11b), which is a typical particle concentration in benthic environment as reported by other studies (Reynolds et al. 2010; Bertuccioli et al. 1999). The VIV relies on natural particles without the need for particle seeding, so it is susceptible to ambient conditions. With the current optical setup, the instrument can only image particles of $50 \mu \mathrm{m}$ or larger, so it needs to operate in environments with sufficient number of particles in this size range, such as coastal areas. To operate in environments with very clear water, such as open ocean (O'Dowd et al. 2010), the instrument will need a more advanced camera system that can image smaller particles.

As an additional constraint, the VIV might not work as well when particle concentrations are particularly high. At higher particle counts per image frame, crowding can cause the adaptive thresholding preprocessing step to group several particles in the same clump, reducing the amount of signal. This would result in a source of the noise in each particle movement, even though all particles might be flowing in the same direction. This noise accumulates in the cross-correlation map, causing the cross-correlation values to be noisy. A more rigorous study of the performance of the algorithm and the VIV with various particle concentrations and sizes would be a valuable adjunct to this work. Nevertheless, the VIV can perform well in typical particle concentrations in coastal environments as shown in the field experiment.

\section{c. Real-time processing}

The current iteration of the VIV utilizes a Raspberry Pi Zero $\mathrm{W}$ because of its low cost and low power consumption,

TABLE 2. Sampling rate and power consumption of single-board computers performing velocity measurement.

\begin{tabular}{lcc}
\hline \hline \multicolumn{1}{c}{ Computer } & $\begin{array}{c}\text { Sampling } \\
\text { rate }(\mathrm{Hz})\end{array}$ & $\begin{array}{c}\text { Power } \\
\text { consumption }(\mathrm{W})\end{array}$ \\
\hline Raspberry Pi Zero W & $0.21 \pm 0.2$ & $1.0 \pm 0.1$ \\
Raspberry Pi 3A+ & $0.99 \pm 0.2$ & $1.5 \pm 0.2$ \\
Raspberry Pi 4B & $1.31 \pm 0.1$ & $2.8 \pm 0.1$ \\
Jetson Nano & $3.76 \pm 0.4$ & $3.1 \pm 0.1$ \\
\hline
\end{tabular}




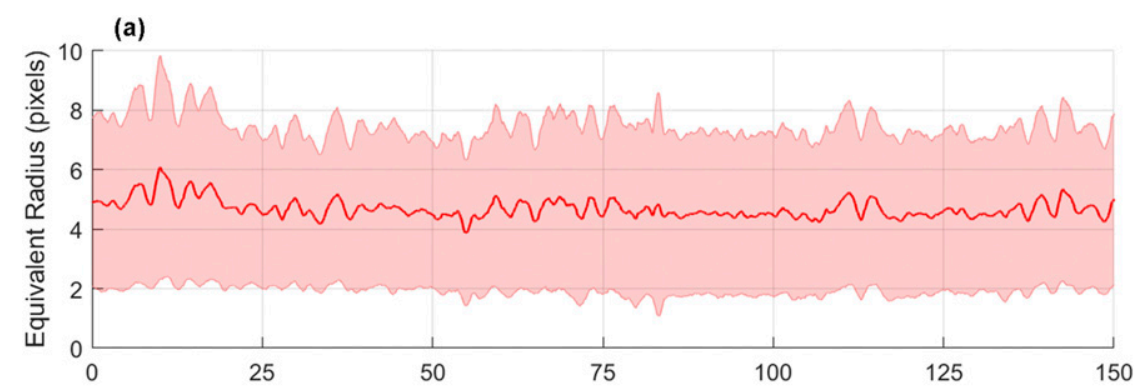

(b)

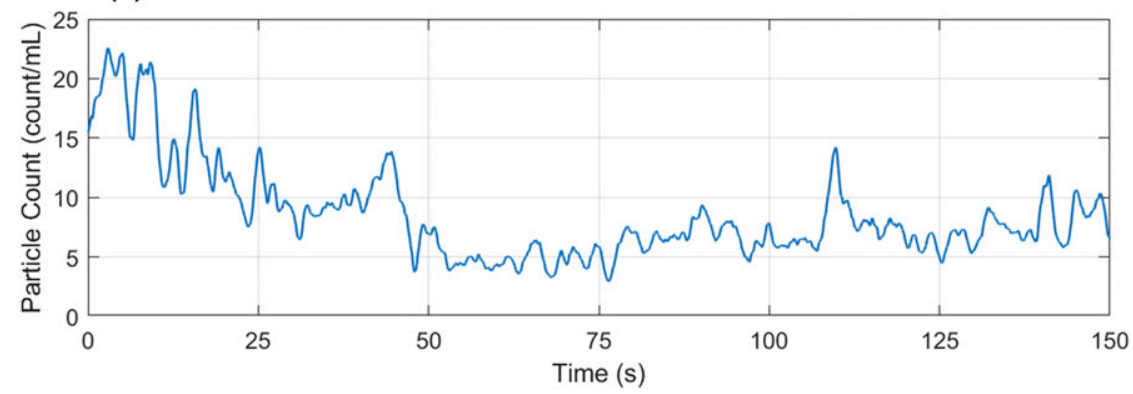

FIG. 11. (a) The time series of equivalent radius of particles detected by the adaptive thresholding process from the field deployment. The line indicates the mean radius in each frame, and the shaded error bar indicates the standard error of the radius. (b) The time series of the particle concentrations from the field deployment.

so it is not suitable for real-time velocity estimation. However, the VIV is designed such that the system is compatible with other more powerful Raspberry Pi computing units, such as Raspberry Pi model 3A + and 4B, that can perform the velocity measurement at approximately 1 sample per second. Even though this sampling rate is not sufficient for small-scale flow that changes rapidly, such as turbulence, it still suits many oceanographic applications that measure medium-scale flow, such as the periodic wave shown in Fig. 8, which has a period of approximately $9 \mathrm{~s}$. These more powerful computing units can readily fit inside the waterproof housing and only require minimal change in the internal mounting.

With more a powerful computer, such as the Jetson Nano, the algorithm can be performed very rapidly and achieve a higher sampling. This high real-time sampling rate indicates that the VIV can be improved with more sophisticated hardware and can be deployed to study flows that change more rapidly.

\section{d. Future improvements}

With the current setup, there are only two views of the sampling volume available to the VIV. In considering further development and application of a more advanced system, future improvement might involve adding more views either by using more complex mirror optics or more cameras. More complex optics and additional views will result in imaging more particles that are in-focus, resulting in cleaner cross-correlation maps. As envisioned, these improvements will also allow the instrument to perform more precise particle correspondence that enables the reconstruction of velocity fields as opposed to a single vector. More powerful computer can also be used to enhance processing such that more complex algorithms, such as tomographic PIV, can be implemented.

Additionally, the VIV is designed to be modular and is based of components that are easily accessible through maker practice. With the rapid advancement in the maker technology, there will be new electronic components, such as cameras and illumination modules, that can improve the performance of the VIV while only require minimal redesign of the system. In addition to being low cost, the VIV is also designed to have low complexity and can be programmed through simple platforms including both Python and Arduino programming. These two platforms are accessible to researchers that do not have extensive electrical engineering training, allowing the VIV to be readily customized based on the specific need of each research study. These advantages will allow the instrument to be used by researchers from many disciplines in oceanography and limnology.

\section{Conclusions}

In this study, we designed, fabricated, and tested an optical system for measuring currents using the ambient distribution of imaged particles to track the flow. The Video Velocimeter (VIV) was developed, calibrated in the laboratory, and then deployed in the field to reconstruct oceanographic currents. As demonstrated, when used in nonturbulent flow, the VIV can estimate mean flow velocity with root-mean-squared errors of the main flow within $10 \%$ of the $\pm 20 \mathrm{~cm} \mathrm{~s}^{-1}$ measurement range when compared to an $\mathrm{ADV}$. 
The key to understand many biological and physical features of the ocean lies in the understanding of the ocean currents that determine the fate of those features. There are still many challenges in measuring the small-scale flow of these currents around complex benthic structure that cannot be accomplished by a single instrument like an ADCP or ADV. The resulting science that is facilitated by having multiple sensors at different locations will surely be enhanced. By developing a new tool that is inexpensive and easy to use by researchers across many disciplines, we hope that the VIV will be a platform that facilitates new discoveries in the underwater environment.

Acknowledgments. The authors gratefully acknowledge the support from Sontek, a Xylem brand, for their loans of the acoustic Doppler velocimeters used throughout this study. The author would also like to thank Devin Ratelle for his support on mechanical development of the instrument and Pochara Lertvilai for his help on the graphics used in this article.

\section{REFERENCES}

Bertuccioli, L., G. I. Roth, J. Katz, and T. R. Osborn, 1999: A submersible particle image velocimetry system for turbulence measurements in the bottom boundary layer. J. Atmos. Oceanic Technol., 16, 1635-1646, https://doi.org/10.1175/15200426(1999)016<1635:ASPIVS>2.0.CO;2.

Bourdon, C. J., M. G. Olsen, and A. D. Gorby, 2003: Validation of an analytical solution for depth of correlation in microscopic particle image velocimetry. Meas. Sci. Technol., 15, 318-327, https://doi.org/10.1088/0957-0233/15/2/002.

Chételat, O., and K. C. Kim, 2002: Miniature particle image velocimetry system with LED in-line illumination. Meas. Sci. Technol., 13, 1006, https://doi.org/10.1088/0957-0233/13/7/306.

_ S. Y. Yoon, and K. C. Kim, 2001: Design and construction of a miniature PIV (MPIV) system. KSME Int. J., 15, 1775-1783, https://doi.org/10.1007/BF03185134.

Davis, C. S., G. R. Flierl, P. H. Wiebe, and P. J. S. Franks, 1991: Micropatchiness, turbulence and recruitment in plankton. J. Mar. Res., 49, 109-151, https://doi.org/10.1357/002224091784968602.

Ekman, V. W., 1932: An improved type of current-meter. ICES J. Mar. Sci., 7, 3-10, https://doi.org/10.1093/icesjms/7.1.3.

Estevadeordal, J., and L. Goss, 2005: PIV with LED: Particle shadow velocimetry (PSV). 43rd AIAA Aerospace Science Meeting and Exhibit, Reno, NV, AIAA, https://doi.org/10.2514/ 6.2005-37.

Fouras, A., D. Lo Jacono, C. V. Nguyen, and K. Hourigan, 2009: Volumetric correlation PIV: A new technique for 3D velocity vector field measurement. Experiments in Fluids, Vol. 47, Springer, 569-577.

Fuentes-Perez, J. F., C. Meurer, J. A. Tuhtan, and M. Kruusmaa, 2018: Differential pressure sensors for underwater speedometry in variable velocity and acceleration conditions. IEEE J. Ocean. Eng., 43, 418-426, https://doi.org/10.1109/JOE.2017.2767786.

Garwood, J. C., A. J. Lucas, P. Naughton, M. H. Alford, P. L. D. Roberts, J. S. Jaffe, L. deGelleke, and P. J. S. Franks, 2020: A novel cross-shore transport mechanism revealed by subsurface, robotic larval mimics: Internal wave deformation of the background velocity field. Limnol. Oceanogr., 65, 1456-1470, https://doi.org/10.1002/lno.11400.

Gaylord, B., and Coauthors, 2007: Spatial patterns of flow and their modification within and around a giant kelp forest. Limnol. Oceanogr., 52, 1838-1852, https://doi.org/10.4319/lo.2007.52.5.1838.
Hagsäter, S. M., C. H. Westergaard, H. Bruus, and J. P. Kutter, 2008: Investigations on LED illumination for micro-PIV including a novel front-lit configuration. Exp. Fluids, 44, 211-219, https://doi.org/10.1007/s00348-007-0394-z.

Hench, J. L., and J. H. Rosman, 2013: Observations of spatial flow patterns at the coral colony scale on a shallow reef flat. J. Geophys. Res. Oceans, 118, 1142-1156, https://doi.org/ 10.1002/jgrc.20105.

Hill, P., A. R. Nowell, and P. Jumars, 1992: Encounter rate by turbulent shear of particles similar in diameter to the Kolmogorov scale. J. Mar. Res., 50, 643-668, https://doi.org/ $10.1357 / 002224092784797539$.

Huang, Z.-C., L. Lenain, W. K. Melville, J. H. Middleton, B. Reineman, N. Statom, and R. M. McCabe, 2012: Dissipation of wave energy and turbulence in a shallow coral reef lagoon. J. Geophys. Res., 117, C03015, https://doi.org/10.1029/ 2011JC007202.

Jaffe, J. S., P. J. S. Franks, P. L. D. Roberts, D. Mirza, C. Schurgers, R. Kastner, and A. Boch, 2017: A swarm of autonomous miniature underwater robot drifters for exploring submesoscale ocean dynamics. Nat. Commun., 8, 14189, https:// doi.org/10.1038/ncomms14189.

Jickells, T. D., 1998: Nutrient biogeochemistry of the coastal zone. Science, 281, 217-222, https://doi.org/10.1126/science.281.5374.217.

Johansen, J. L., 2014: Quantifying water flow within aquatic ecosystems using load cell sensors: A profile of currents experienced by coral reef organisms around Lizard Island, Great Barrier Reef, Australia. PLOS ONE, 9, e83240, https:// doi.org/10.1371/journal.pone.0083240.

Jones, I. S. F., 1980: Electromagnetic current meters. Air-Sea Interaction, Springer, 219-229.

Katija, K., and J. O. Dabiri, 2008: In situ field measurements of aquatic animal-fluid interactions using a self-contained underwater velocimetry apparatus (SCUVA). Limnol. Oceanogr. Methods, 6, 162-171, https://doi.org/10.4319/lom.2008.6.162.

Knauss, J. A., 1959: Measurements of the Cromwell Current. DeepSea Res., 6, 265-274, https://doi.org/10.1016/0146-6313(59) 90086-3.

Lacoursière, J. O., and D. A. Craig, 1990: A small flume for studying the influence of hydrodynamic factors on benthic invertebrate behaviour. J. North Amer. Benthological Soc., 9, 358-367, https://doi.org/10.2307/1467903.

Liao, Q., H. A. Bootsma, J. Xiao, J. V. Klump, A. Hume, M. H. Long, and P. Berg, 2009: Development of an in situ underwater particle image velocimetry (UWPIV) system. Limnol. Oceanogr. Methods, 7, 169-184, https://doi.org/10.4319/lom.2009.7.169.

Liberzon, A., D. Lasagna, M. Aubert, P. Bachant, and J. Borg, 2016: Openpiv/Openpiv-Python: Updated Pyprocess with extended area search method. ePrints Soton, accessed 19 May 2020, https://eprints.soton.ac.uk/434472/.

Lohrmann, A., R. Cabrera, and N. C. Kraus, 1994: AcousticDoppler velocimeter (ADV) for laboratory use. Proc. Symp. on Hydraulic Measurements and Experimentation, Buffalo, NY, ASCE, 351-365.

Mach, K. J., S. K. Tepler, A. V. Staaf, J. C. Bohnhoff, and M. W. Denny, 2011: Failure by fatigue in the field: A model of fatigue breakage for the macroalga Mazzaella, with validation. J. Exp. Biol., 214, 1571-1585, https://doi.org/10.1242/jeb.051623.

MacVicar, B. J., E. Beaulieu, V. Champagne, and A. G. Roy, 2007: Measuring water velocity in highly turbulent flows: Field tests of an electromagnetic current meter (ECM) and an acoustic Doppler velocimeter (ADV). Earth Surf. Processes Landforms, 32, 1412-1432, https://doi.org/10.1002/esp.1497. 
Madin, J. S., and S. R. Connolly, 2006: Ecological consequences of major hydrodynamic disturbances on coral reefs. Nature, 444, 477-480, https://doi.org/10.1038/nature05328.

Martin, A. P., and K. J. Richards, 2001: Mechanisms for vertical nutrient transport within a North Atlantic mesoscale eddy. Deep-Sea Res. II, 48, 757-773, https://doi.org/10.1016/S09670645(00)00096-5.

Meinhart, C. D., S. T. Wereley, and J. G. Santiago, 2000: A PIV algorithm for estimating time-averaged velocity fields. $J$. Fluids Eng., 122, 285-289, https://doi.org/10.1115/1.483256.

O'Dowd, C., C. Monahan, and M. Dall'Osto, 2010: On the occurrence of open ocean particle production and growth events. Geophys. Res. Lett., 37, L19805, https://doi.org/10.1029/2010GL044679.

Olsen, M. G., and R. J. Adrian, 2000: Out-of-focus effects on particle image visibility and correlation in microscopic particle image velocimetry. Experiments in Fluids, Vol. 29, Springer, S166-S174.

Pagnutti, M. A., R. E. Ryan, G. J. Cazenavette, M. J. Gold, R. Harlan, E. Leggett, and J. F. Pagnutti, 2017: Laying the foundation to use Raspberry Pi 3 V2 camera module imagery for scientific and engineering purposes. J. Electron. Imaging, 26, 013014, https://doi.org/10.1117/1.JEI.26.1.013014.

Pineda, J., J. Hare, and S. Sponaugle, 2011: Larval transport and dispersal in the coastal ocean and consequences for population connectivity. Oceanography, 20 (3), 22-39, https://doi.org/ 10.5670/oceanog.2007.27.

Prasad, A. K., 2000: Stereoscopic particle image velocimetry. Exp. Fluids, 29, 103-116, https://doi.org/10.1007/s003480000143.

—, 2007: Particle image velocimetry. Curr. Sci., 79, 51-60.

Raffel, M., C. E. Willert, S. T. Wereley, and J. Kompenhans, 1989: Particle Image Velocimetry: A Practical Guide. Springer, 30 pp.

Reynolds, R. A., D. Stramski, V. M. Wright, and S. B. Woźniak, 2010: Measurements and characterization of particle size distributions in coastal waters. J. Geophys. Res., 115, C08024, https://doi.org/10.1029/2009JC005930.

Rogers, J. S., S. A. Maticka, V. Chirayath, C. B. Woodson, J. J. Alonso, and S. G. Monismith, 2018: Connecting flow over complex terrain to hydrodynamic roughness on a coral reef. J. Phys. Oceanogr., 48, 1567-1587, https://doi.org/10.1175/ JPO-D-18-0013.1.
Rothschild, B. J., and T. R. Osborn, 1988: Small-scale turbulence and plankton contact rates. J. Plankton Res., 10, 465-474, https://doi.org/10.1093/plankt/10.3.465.

Rudnick, D. L., R. E. Davis, C. C. Eriksen, D. M. Fratantoni, and M. J. Perry, 2004: Underwater gliders for ocean research. Mar. Technol. Soc. J., 38, 73-84, https://doi.org/ 10.4031/002533204787522703.

Smith, W. A. M. N., 2008: A submersible three-dimensional particle tracking velocimetry system for flow visualization in the coastal ocean. Limnol. Oceanogr. Methods, 6, 96-104, https:// doi.org/10.4319/lom.2008.6.96.

Steinbuck, J. V., and Coauthors, 2010: An autonomous open-ocean stereoscopic PIV profiler. J. Atmos. Oceanic Technol., 27, 1362-1380, https://doi.org/10.1175/2010JTECHO694.1.

Tanenbaum, J. G., A. M. Williams, A. Desjardins, and K. Tanenbaum, 2013: Democratizing technology: Pleasure, utility and expressiveness in DIY and maker practice. Proc. Conf. on Human Factors in Computing Systems, New York, NY, ACM, 2603-2612.

Tritico, H. M., A. J. Cotel, and J. N. Clarke, 2007: Development, testing and demonstration of a portable submersible miniature particle imaging velocimetry device. Meas. Sci. Technol., 18, 2555-2562, https://doi.org/10.1088/0957-0233/18/8/031.

Weller, R. A., and R. E. Davis, 1980: A vector measuring current meter. Deep-Sea Res., 27A, 565-581, https://doi.org/10.1016/ 0198-0149(80)90041-2.

Wellner, P. D., 1993: Adaptive thresholding for the DigitalDesk. EuroPARC Tech. Rep. EPC-93-110, 14 pp.

Xue, Z., J. J. Charonko, and P. P. Vlachos, 2014: Particle image velocimetry correlation signal-to-noise ratio metrics and measurement uncertainty quantification. Meas. Sci. Technol., 25, 115301, https://doi.org/10.1088/0957-0233/25/11/115301.

Yu, L., and B. Pan, 2016: Structure parameter analysis and uncertainty evaluation for single-camera stereo-digital image correlation with a four-mirror adapter. Appl. Opt., 55, 6936, https://doi.org/10.1364/AO.55.006936.

Zhang, Z., 1999: Flexible camera calibration by viewing a plane from unknown orientations. Proc. Seventh IEEE Int. Conf. on Computer Vision, Kerkyra, Greece, IEEE, 666-673, https:// doi.org/10.1109/ICCV.1999.791289. 